\title{
Technical note \\ Development of a gradient tube method for examining microbial population structures in floating sulphur biofilms
}

\author{
ML Bowker1, JB Molwantwa², J Gilfillan³, R Dorrington³, R Kirby4 and PD Rose ${ }^{3 *}$ \\ 'Environmental Biotechnology Research Institute, Rhodes University, Grahamstown, South Africa \\ ${ }^{2}$ Digby Wells Associates, Johannesburg, South Africa \\ ${ }^{3}$ Department of Biochemistry, Microbiology and Biotechnology, Rhodes University, Grahamstown, South Africa \\ ${ }^{4}$ Department of Life Sciences and Institute of Genome Science, National Yang-Ming University, Taiwan
}

\begin{abstract}
Floating biofilms occur in thin layers of between $50 \mu \mathrm{m}$ and $500 \mu \mathrm{m}$ on the surface of certain organic, sulphidic aquatic environments and, at times, may only be several cells deep. While these structures may be important in terms of energy flow pathways, and possibly also in wastewater treatment operations, little is known about their structural/functional properties. This is due, in part, to their flimsy nature but also to methodological constraints related to their sampling and manipulation. We have investigated floating sulphur biofilms that appear as white layers on the surface of anoxic sulphidic organic wastewaters and describe here the development of a novel gradient tube method for investigating these systems. This approach enables testing of the hypothesis that these floating sulphur biofilms are complex well-differentiated structures rather than disordered dispersions of microbial biomass as has been previously thought. Furthermore, if the former is correct, they would seem to resemble the structure and functionality of comparable complex bioflms that are attached to solid substrates. The gradient tube method involves the establishment of apposing gradients of sulphide and oxygen that are expanded across a tube of agarose $10 \mathrm{~cm}$ in length; this simulates the oxic/anoxic interface that occurs over only several micrometres in the natural biofilm system. A plug of sulphide-enriched agarose is first placed in the base of the tube. Samples of the floating sulphur biofilm are then mixed into agarose growth medium and, before it sets, this is overlaid on top of the plug. The tubes are then open capped and incubated. A variety of different microbial populations may thus become established in the separate physiological niches that are set up in this way within the gradient tube. The populations may be quite robustly sampled by extruding and then sectioning the agarose plug. This expansion of the biofilm enables more detailed molecular phylogenetic studies of the populations found in the various niches within the biofilm and also measurement of physico-chemical parameters within the system.
\end{abstract}

Keywords: gradient tube method, floating biofilms, floating sulphur biofilms, microbial ecology, sulphur biotechnology, acid mine drainage wastewaters

\section{INTRODUCTION}

The examination of the population structure of biofilms, and particularly attempts to correlate structure and function in a way that rigorously represents the actual situation within the biofilm, remains an exacting methodological task. The requirements are particularly acute when studying floating biofilms that occur as layers of around $50 \mu \mathrm{m}$ to $500 \mu \mathrm{m}$ on water surfaces in some aquatic environments and are, during the early stages of formation, possibly only several cells deep (Gilfillan, 2000).

We have examined floating sulphur biofilms that form at the oxic/anoxic interface on the surface of tannery evaporation ponds (Fig. 1A), which receive high organic and sulphide loads (Gilfillan, 2000; Bowker, 2002). Similar structures have been observed in other sulphidic aquatic environments such as brackish and salt marshes and these biofilms may be an important component in total energy flow pathways in these systems (Whitcomb, 1989). While it has been suggested that they are simple accumulations of microbial growth, we have

\footnotetext{
To whom all correspondence should be addressed.

IIII)+2782 8101353; e-mail: p.rose@ru.ac.za

Received 23 July 2012; accepted in revised form 24 June 2013.
}

found that these floating sulphur biofilms are composed of both obligate aerobic and anaerobic populations, including sulphide oxidising bacteria, which suggests rather that they are complex well-differentiated structures that are comparable to stratified biofilm growth found on solid substrates (Monds and O'Toole, 2009). While scanning electron microscopy of the floating sulphur biofilm (Fig. 2) clearly shows that the microbial population is embedded in an exopolysaccharide matrix that includes the production of ortho-rhombic crystals of elemental sulphur (Bowker, 2002), the possible functional differentiation of the population structure within the biofilm has remained undescribed up to the present.

The possible application of the floating sulphur biofilm system for sulphur recovery in the biological treatment of acid mine drainage wastewaters has been proposed and bioprocess development studies have been reported by Gilfillan (2000), Bowker (2002), Bowker et al. (2002), Molwantwa et al. (2007), Molwantwa (2008), Rose and Rein (2007), Molwantwa et al. (2009), Mack et al. (2009) and Van Hille et al. (2011). However, uncertainty about a biological role, if any, for sulphur formation in these films, the possible presence of differentiation within the microbial population structure and, importantly, the possibility of physiological functional differentiation within this type of system has remained unresolved. The main reasons for this are methodological constraints related to sampling and 

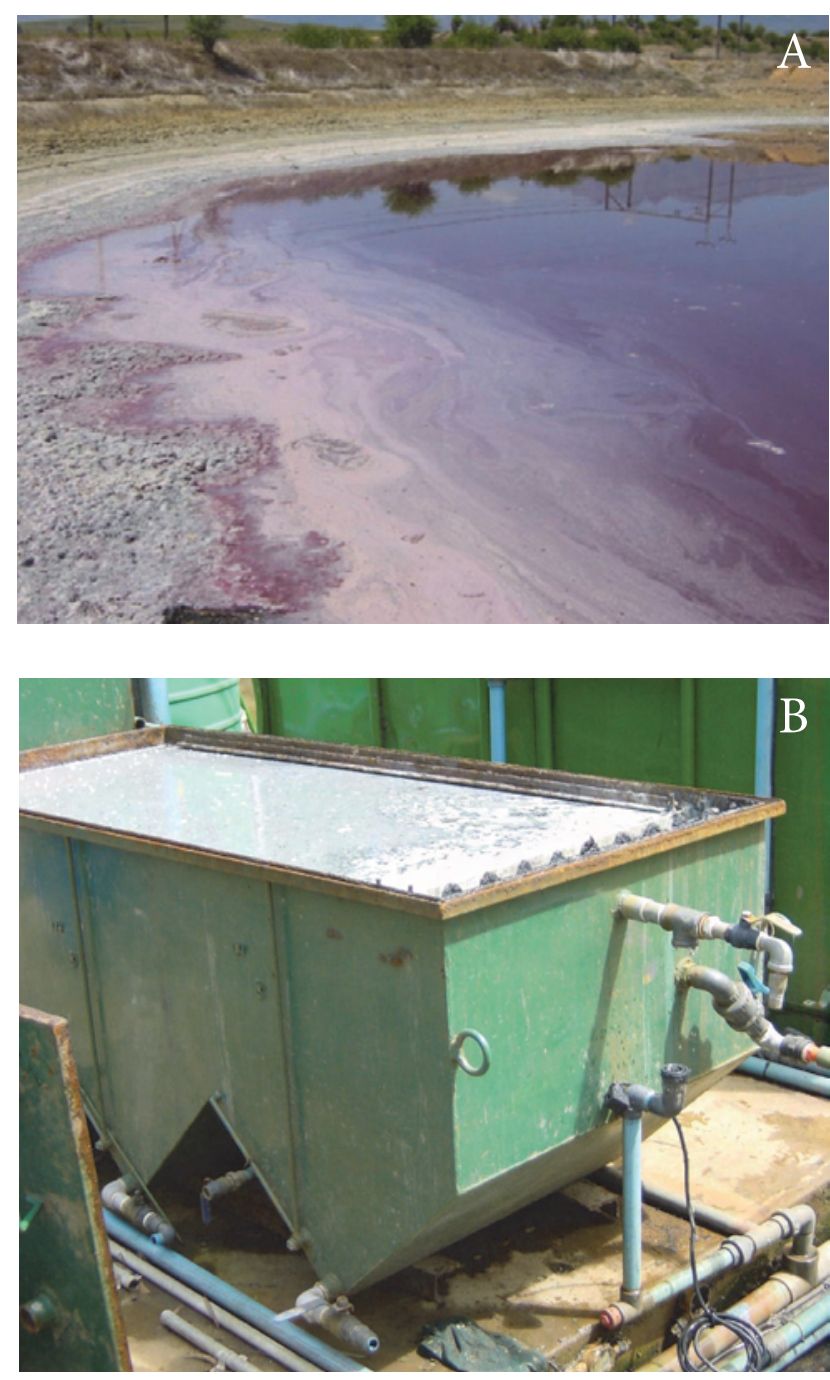

Figure 1

A. Photograph of a tannery wastewater evaporation pond, an example of a high-sulphide organic-enriched aquatic environment, and showing formation of the white sulphur biofilm floating on the water surface. Sulphur produced in this system is blown into thick windrows on the sides of the levies but may also be deposited as a sediment within the pond.

B. Field-scale floating sulphur biofilm reactor developed to enable the simulation of floating sulphur biofilm formation under experimental conditions.

experimental manipulation of these thin and fragile structures. More clarity on the structural/functional attributes of these floating biofilm systems could be important to the future engineering of their use in wastewater treatment applications. We report here the development of a gradient tube method for expanding the spatial distribution of the various physiologically functional microbial groups that grow actively within these biofilms. This approach allows the effective sampling of the different components found within the biofilm population structure.

\section{MATERIALS AND METHODS}

Actively growing floating sulphur biofilm was sampled from both tannery ponding systems (Fig. 1A), and a laboratory reactor (Fig.1B) that was fed organic sulphide-enriched water generated in a degrading packed bed reactor as described by Molwantwa et al. (2007). Aliquots of these

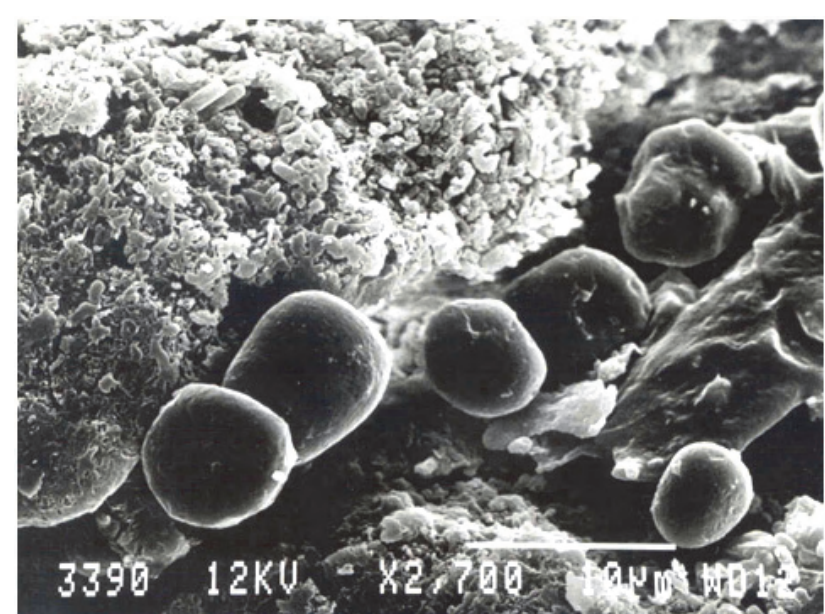

Figure 2

Scanning electron micrograph showing large amorphous structures which accumulate on the underside of the floating sulphur biofilm. These were shown elsewhere to be composed largely of sulphur, possibly in the long chain polysulphide form.

biofilms $(500 \mu \ell)$ were mixed with $500 \mu \ell$ of glycerol and frozen at $-70^{\circ} \mathrm{C}$ until used.

Gradient tubes were prepared by aseptically placing, at the bottom of a sterile $15 \mathrm{~m} \ell$ glass test tube, a $5 \mathrm{~m} \ell$ molten agarose plug containing $1 \%$ low-melt agarose, $0.5 \mathrm{~g} / \ell$ sodium sulphide in lactate medium ( $1.75 \mathrm{~m} \ell 60 \%$ sodium lactate, $1 \mathrm{~g} \mathrm{MgSO}_{4} \cdot 7 \mathrm{H}_{2} \mathrm{O}, 0.5 \mathrm{~g}$ yeast extract, $0.25 \mathrm{~g} \mathrm{~K}_{2} \mathrm{HPO}_{4}, 0.05 \mathrm{~g}$ $\mathrm{CaCl}_{2} \cdot 6 \mathrm{H}_{2} \mathrm{O}, 0.5 \mathrm{~g} \mathrm{NH}_{4} \mathrm{Cl}$; made up in $250 \mathrm{~m} \ell$ with ddd $\mathrm{H}_{2} \mathrm{O}$ and sterilised). After the sulphide plug had set, an overlay of the same lactate medium, but without sodium sulphide, was prepared, to which the biofilm sample was added and thoroughly mixed; the biofilm inoculum was added while the medium was still liquid but as cool as possible. Then $10 \mathrm{~m} \ell$ of the mixture was pipetted aseptically onto the surface of the solid agarose sulphide plug while avoiding the introduction of air bubbles. The tubes were open capped (agarose surface having access to air) and incubated in the dark at $20^{\circ} \mathrm{C}$ for 7 days (Fig. 3).

After incubation had been completed, tubes were swabbed with ethanol, etched with a diamond glass cutter and then broken cleanly at the bottom. The agarose plug was then extruded onto an aseptic surface in a laminar flow hood. The plug was sliced into $5 \mathrm{~mm}$ lengths and each sample placed into a separate sterile Eppendorf tube that contained $1 \mathrm{~m} \ell$ sterile Tris-EDTA buffer (0.01 M Tris, 0.05M EDTA). Samples were heated to $50^{\circ} \mathrm{C}$, vortexed, and (with the exception of those to be used in sulphide analyses) were stored at $4^{\circ} \mathrm{C}$ overnight to facilitate diffusion. They were then analysed to measure the levels of sulphate, sulphur and total organic carbon (TOC). Samples for molecular phylogenetic analysis (results not reported here) were obtained prior to the heating step.

For the sulphide analysis, $20 \mu \ell$ samples were taken immediately from each of the $0.5 \mathrm{~g}$ sections and added to $100 \mu \ell$ of zinc acetate and made up to $5 \mathrm{~m} \ell$ with ddd $\mathrm{H}_{2} \mathrm{O}$. The assay was performed as follows: the samples were vortexed briefly and $500 \mu \ell$ of ferric chloride solution $\left(8 \mathrm{~g} \mathrm{FeCl}_{3}\right.$ in $500 \mathrm{m \ell}$ $6 \mathrm{M} \mathrm{HCL})$ and $500 \mu \ell$ amide-sulphuric acid solution $(2 \mathrm{~g} \mathrm{~N}-\mathrm{N}$ dimethyl-p-phenylene diamine dihydrochloride in $500 \mathrm{~m} \ell$ $6 \mathrm{M} \mathrm{H}_{2} \mathrm{SO}_{4}$ ) were added. The samples were allowed to stand at room temperature for $1 \mathrm{~h}$ and then the absorbance was read on a Shimadzu UV-160A UV-visible recording spectrophotometer at $670 \mathrm{~nm}$. 
For sulphur analysis $200 \mu \ell$ of each $0.5 \mathrm{~g}$ sample was placed in an Eppendorf tube and microfuged at $13000 \mathrm{r} / \mathrm{min}$ for 10 min. The supernatant was discarded and the pellet re-suspended in $100 \mu \ell$ of high performance liquid chromatography (HPLC) grade acetone. The sample was allowed to stand for

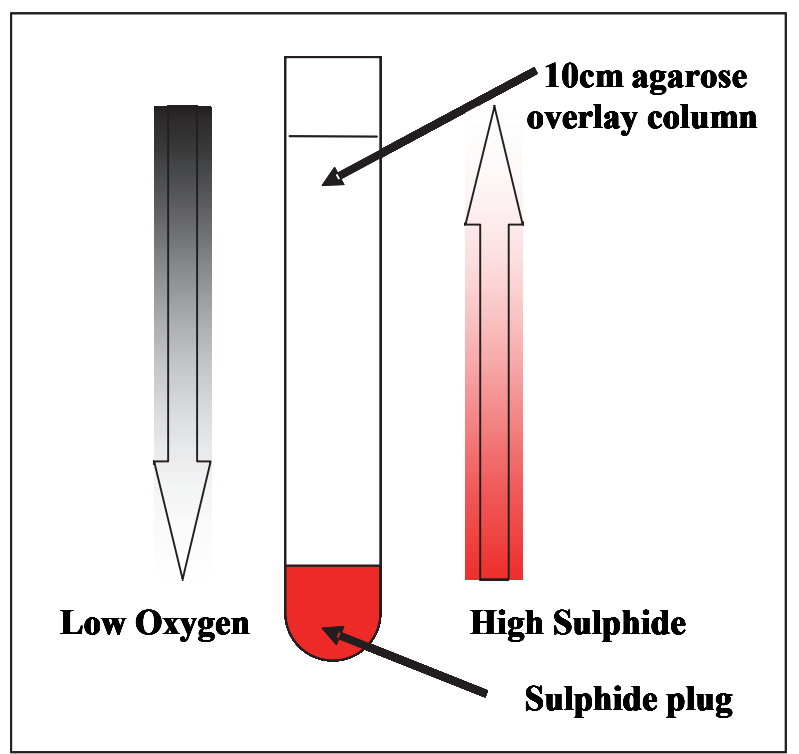

Figure 3

Diagram showing the setup of the gradient tubes with a plug of sulphide in the base and overlaid with agarose in which a composite sample of biofilm had been suspended. The opposing gradients of oxygen and sulphide that are set up in this way are shown.
$1 \mathrm{~h}$ and then filtered through a $0.45 \mu \mathrm{m}$ nylon membrane to remove any particulate matter. The samples were analysed by HPLC using a Phenomenex LUNA $5 \mu$ C18 (2) size 150 x $4.6 \mathrm{~mm}$ column, mobile phase 95:5 methanol:water, run at a flow rate of $2 \mathrm{ml} / \mathrm{min}$ and using a Waters 484 Absorbance Detector.

Sulphate analysis was performed by HPLC. Samples were diluted 1 in 10 and then $1 \mathrm{~m} \ell$ of this sample was filtered through a $0.45 \mu \mathrm{m}$ nylon filter followed by a Waters C18 solidphase extraction cartridge. A Hamilton PRP-X100 $10 \mu \mathrm{m} 150$ $\mathrm{x} 4.1 \mathrm{~mm}$ column with a para-hydroxybenzoic acid/methanol (5.52 g:250 $\mathrm{m} \ell$ ) mobile phase was used at a flow rate of $2 \mathrm{m \ell} /$ min with a Waters 430 conductivity detector.

For total organic carbon (TOC) analysis, a ROSEMOUNT Dohrmann Total Organic Carbon Analyzer DC-180 was used. All reagents were supplied by Merck.

\section{RESULTS AND DISCUSSSION}

Figure 3 illustrates the principle of the gradient tube method whereby apposing gradients, in reducing concentrations, are established, and where sulphide migrates from the bottom plug upwards and oxygen migrates from the top of the tube downwards. This is designed to replicate conditions at the oxic/ anoxic interface of the water surface in the sulphidic aquatic environments being investigated, where oxygen migrating from the air into the water column meets the underlying sulphide layer. It is in the presence of this type of gradient that the appearance of the floating sulphur biofilm structures is observed. In the tube method, gradients of high sulphide/low oxygen and low sulphide/high oxygen are established over a $10 \mathrm{~cm}$ length of the agarose plug; this provides a range of niches for the growth of any physiologically distinct microbial populations that are present in the original biofilm. These would possibly grow and be discernible as separate zones of population dominance within the tube, which has now been expanded across the $10 \mathrm{~cm}$ length of the agarose column, rather than the $50 \mu \mathrm{m}$ to $500 \mu \mathrm{m}$ of the original biofilm.

The results detailed in Fig. 4 report analyses for sulphide, sulphur, sulphate and TOC for the $5 \mathrm{~mm}$

\section{Figure 4}

A. Sulphide concentration profile through the depth of the gradient tube as measured in each of the $5 \mathrm{~mm}$ agarose cut sections. An inoculated tube is compared with the uninoculated control.

B. Sulphur concentration profile through the depth of the gradient tube as measured in each of the $5 \mathrm{~mm}$ agarose cut sections. An inoculated tube is compared with the uninoculated control.

C. Sulphate concentration profile through the depth of the gradient tube as measured in each of the $5 \mathrm{~mm}$ agarose cut sections. An inoculated tube is compared with the uninoculated control.

D. Total organic carbon concentration profile through the depth of the gradient tube as measured in each of the $5 \mathrm{~mm}$ agarose cut sections. An inoculated tube is compared with the uninoculated control. 

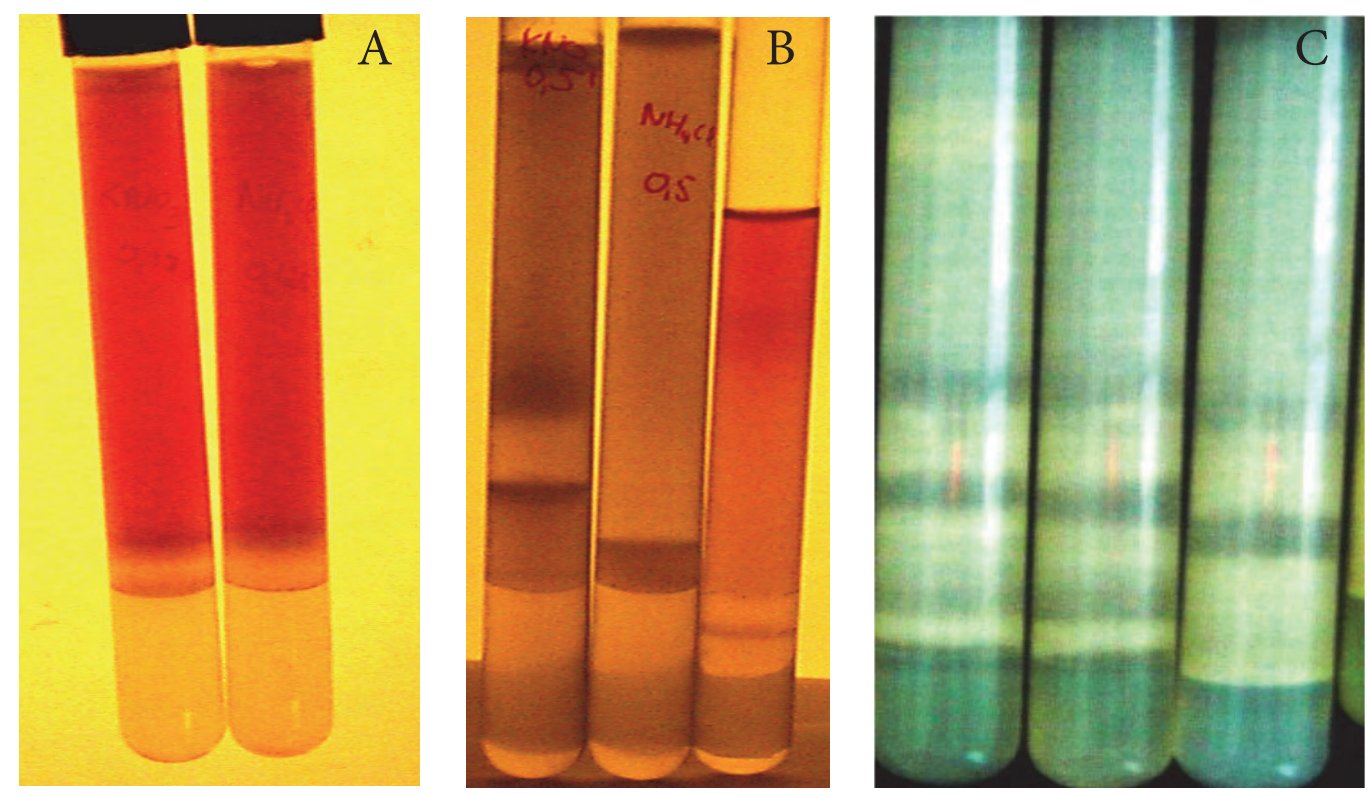

Figure 5

Photographs of uninoculated gradient tube controls (A) and inoculated tubes (B) showing the appearance of visible bands and a change in colour of the neutral red indicator after $24 \mathrm{~h}$ incubation. The sulphide plug may be seen in the base of the tube. Photograph of inoculated tubes after incubation for 7 days showing white bands of sulphur forming at different levels within the gradient tubes (C).

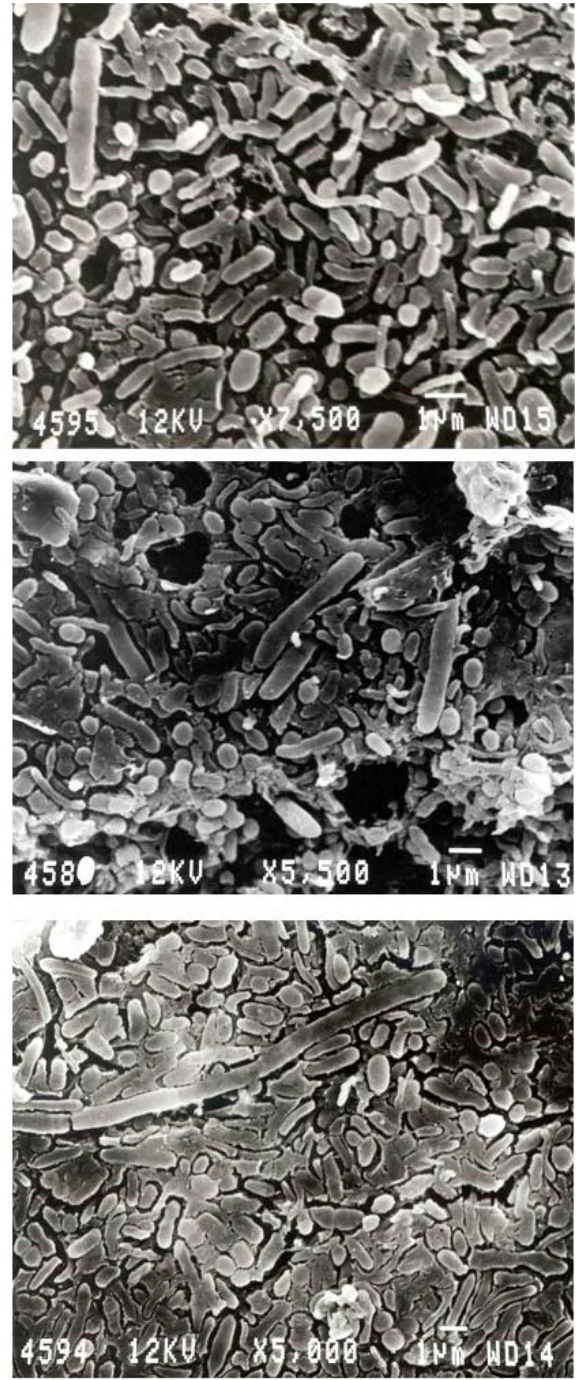

Figure 6

Scanning electron micrograph of inoculated agarose samples from the top (A), middle (B) and bottom (C) sections of separate gradient tubes. The presence of different microbial morphological types occurring at different levels is apparent, with coccoid forms predominating in the upper layers, rods in the middle layers and filamentous forms appearing also in the bottom layers. agarose column cut sections, and compare various biofilm inoculated samples with uninoculated controls. These results are plotted as depth profiles on the y-axis and report the results within the agarose column from the bottom of the tube upwards, which occurs against a background of increasing oxygen and decreasing sulphide concentrations.

Comparison of controls against experimental results reported in Fig. 4 shows that a sulphide gradient is established along the length of the agarose column as anticipated. In the biofilminoculated sample sulphide removal was found to be active across about $80 \%$ of the column length. Figures $4 \mathrm{~B}$ and $4 \mathrm{C}$ report the sulphur and sulphate concentration profiles, respectively. These results indicate the presence of incomplete oxidation of sulphide with a predominant conversion to sulphur, rather than complete oxidation to sulphate. These findings indicate a careful poising of the redox environment (Rein 2002) which, if unregulated in some way, would expect to show the complete oxidation of sulphide in the upper oxic zones of the tubes. If a metabolic energy input is required to maintain the redox poising of the system, some TOC would be expected to be consumed in the process, and this is what was found in Fig. 4D, where up to $30 \%$ of TOC was removed in the upper layer of the tubes. 
Figures $5 \mathrm{~B}$ and $5 \mathrm{C}$ show the appearance of sulphur bands in the tubes after 1 day and 7 days of growth, respectively. Figure 6 shows scanning electron micrographs of samples from the top, middle and bottom of the gradient tubes and indicates differentiation of the microbial populations within separate bands. The upper layers consist mainly of cocci, while mainly bacilli and filamentous microorganisms occur in the middle and lower layers. Molecular phylogenetic analyses of the actual population structures established by this method were undertaken and are to be reported elsewhere.

This method can be used successfully to investigate many aspects of the complex population structure of floating sulphur biofilms. However, there are limitations that need to be taken into account. The most important relates to a long-standing problem in microbial ecology studies, highlighted by Amann et al. (1995) and others, that only those groups present in the biofilm that are able to grow under the conditions established in the gradient tube experimental system will be easily identified and therefore studied. These are the organisms used to present any descriptive account of the system. Other organisms present in the biofilm that are unable to grown in the medium present in the gradient tube will go unnoticed and this may bias any conclusions drawn.

\section{CONCLUSIONS}

The gradient tube method is a novel application that enables the study of microbial population structure and function in thin biofilm layers and does this by expanding the size of sample over which experimental investigation is possible.

The results show that gradients are effectively established in this system with respect to sulphide, sulphur and TOC and to a lesser extent also sulphate.

Organisms of different morphological types were observed at different points within the gradients that were established along the tube length.

Follow-up molecular phylogenetic studies of the microbial populations in floating sulphur biofilms, and correlation of these results with biofilm structure, as determined by microprobe measurements, have been undertaken and are to be reported elsewhere.

The gradient tube method provides an instrument that may be used in conjunction with other methodological approaches to throw light on the microbial ecology of floating sulphur biofilm systems. A better understanding of these structures may facilitate their development in bioprocess applications including acid mine drainage wastewater treatment.

\section{REFERENCES}

AMANN R, LUDWIG W and SCHLEIFER K-H (1995) Phylogenetic identification and in situ detection of individual microbial cells without cultivation. Microbiol. Rev. 59 143-169.

BOWKER M (2002) The biology and molecular ecology of floating sulphur biofilms. PhD thesis, Rhodes University, Grahamstown.

BOWKER M, MOLWANTWA JB and ROSE PD (2002) The molecular microbial ecology of floating sulphur biofilms. Proc. WISA Biennial Conference, 19-23 May 2002, Durban.

GILFILLAN J (2000) The structure and microbiology of floating sulphide oxidizing biofilms. Masters Thesis, Rhodes University, Grahamstown.

MACK CL, RAJA SA, PULLES WB, MUHLBAUER RC and HEATH RA (2009) Application of the linear flow channel reactor (LFCR) for the removal of sulphide from semi-treated acid mine drainage (AMD). Abstracts of the International Mine Water Conference, 19-23 October 2009, Pretoria.

MOLWANTWA JB, COETSER SE, HEATH R and ROSE PD (2007) Development of the floating sulphur biofilm reactor for sulphide oxidation in biological water treatment systems. Water SA 30 (5) 655-657.

MOLWANTWA JB (2008) Floating sulphur biofilms: Structure, function and biotechnology. PhD Thesis, Rhodes University, Grahamstown.

MOLWANTWA JB, BOWKER M, GILFILLAN J, REIN R, DORRINGTON R, HART OO and ROSE PD (2009) Investigation and development of the biotechnology of sulphur biofilms in the beneficiation and treatment of wastewater. WRC Report No. TT 411/09. Water Research Commission, Pretoria.

MONDS RD and O'TOOLE GA (2009) The developmental model of microbial biofilms: ten years of a paradigm up for review. Trends Microbiol. 17 (2) 73-87.

ROSE PD and REIN NB (2007) Water Treatment. US Patent 7285216.

VAN HILLE RP, VAN WYK N, MOTLELENG L and MOORUTH N (2011) Lessons in passive treatment: Towards efficient operation of a sulphate reduction - sulphide oxidation system. Abstracts of the International Mine Water Conference, 4-11 September 2011, Aachen, Germany.

WHITCOMB JH, DELAUNE RD and PATRICK JR (1989) Chemical oxidation of sulfide to elemental sulphur: its possible role in marsh energy flow. Mar. Chem. 26 205-214. 
http://dx.doi.org/10.4314/wsa.v39i4.11 Available on website http://www.wrc.org.za

ISSN 0378-4738 (Print) = Water SA Vol. 39 No. 4 July 2013 ISSN 1816-7950 (On-line) = Water SA Vol. 39 No. 4 July 2013 